\title{
Baseline characteristics and outcome for aneurysmal versus non-aneurysmal subarachnoid hemorrhage: a prospective cohort study
}

\author{
Catharina Conzen ${ }^{1}$ (D) Miriam Weiss ${ }^{1} \cdot$ Walid Albanna ${ }^{1} \cdot$ Katharina Seyfried $^{1} \cdot$ Tobias P. Schmidt $^{1}$. \\ Omid Nikoubashman ${ }^{2} \cdot$ Christian Stoppe $^{3} \cdot$ Hans Clusmann $^{1} \cdot$ Gerrit A. Schubert ${ }^{1,4}$
}

Received: 18 May 2021 / Revised: 24 July 2021 / Accepted: 20 September 2021 / Published online: 4 October 2021

(c) The Author(s) 2021

\begin{abstract}
This study aims to investigate the characteristics of patients with mild aneurysmal and non-aneurysmal perimesencephalic and non-perimesencephalic subarachnoid hemorrhage (aSAH, pmSAH, npmSAH) with emphasis on admission biomarkers, clinical course, and outcome. A prospective cohort of 115 patients with aSAH (Hunt and Hess 1-3) and of 35 patients without aneurysms (16 pmSAH and 19 npmSAH) admitted between January 2014 and January 2020 was included. Demographic data, blood samples on admission, complications (hydrocephalus, shunt dependency, delayed cerebral ischemia DCI, DCIrelated infarction, and mortality), and outcome after 6 months were analyzed. Demographic data was comparable between all groups except for age (aSAH 55 [48-65] vs. npmSAH 60 [56-68] vs. pmSAH 52 [42-60], $p=0.032$ ) and loss of consciousness ( $33 \%$ vs. $0 \%$ vs. $0 \%, p=0.0004$ ). Admission biomarkers showed poorer renal function and highest glucose levels for npmSAH patients. Complication rate in npmSAH was high and comparable to that of aSAH patients (hydrocephalus, shunt dependency, DCI, DCI-related infarction, mortality), but nearly absent in patients with pmSAH. Favorable outcome after 6 months was seen in $92.9 \%$ of pmSAH, $83.3 \%$ of npmSAH, and $62.7 \%$ of aSAH $(p=0.0264)$. In this prospective cohort of SAH patients, npmSAH was associated with a complicated clinical course, comparable to that of patients with aSAH. In contrast, such complications were nearly absent in pmSAH patients, suggesting fundamental differences in the pathophysiology of patients with different types of non-aneurysmal hemorrhage. Our findings underline the importance for a precise terminology according the hemorrhage etiology as a basis for more vigilant management of npmSAH patients. NCT02142166, 05/20/2014, retrospectively registered.
\end{abstract}

Keywords Subarachnoid hemorrhage $\cdot$ Good grade $\cdot$ Perimesencephalic $\cdot$ Angiographically negative $\cdot$ Nonperimesencephalic · Delayed cerebral ischemia

Catharina Conzen

cconzen@ukaachen.de

1 Department of Neurosurgery, University Hospital Aachen, RWTH Aachen University, Pauwelsstrasse 30, 52074 Aachen, Germany

2 Department of Neuroradiology, University Hospital Aachen, RWTH Aachen University, Aachen, Germany

3 Department of Intensive Care and Intermediate Care, University Hospital Aachen, RWTH Aachen University, Aachen, Germany

4 Department of Neurosurgery, Kantonsspital Aarau, Aarau, Switzerland

\section{Introduction}

In up to $15 \%$ of patients with spontaneous subarachnoid hemorrhage (SAH), no aneurysm as a source of hemorrhage can be identified [20,24]. The underlying pathophysiology of non-aneurysmal SAH remains poorly understood, but previous studies implicated aberrant venous drainage patterns in the basal cisterns and thereby a low-pressure hemorrhage $[3,21]$. According to the blood distribution on the initial CT scan, non-aneurysmal hemorrhages can be divided into perimesencephalic SAH (pmSAH, blood within the interpeduncular, ambient, and chiasmatic cistern) and non-perimesencephalic SAH (npmSAH, diffuse distribution of blood) $[13,20,23]$. The clinical course and outcomes of patients with pmSAH and npmSAH are commonly reported as rather 
benign, in particular in comparison with aSAH $[6,10,13]$. However, the assumption that patients suffering from SAH without evidence of an aneurysm do not require intensive neurological monitoring during the early stage of admission is increasingly challenged $[1,12,19]$, but robust prospective data are scarce. Further, differences in the pathogenesis, clinical management, and outcomes across these three entities, i.e., aSAH, pmSAH, and npmSAH, have been seldom investigated in a balanced, prospective cohort. This study aims to characterize differences in the clinical course and neurological outcome across different types of SAH.

\section{Materials and methods}

\section{Study design}

This study was approved by the local ethics committee of the University Hospital of Aachen (Ethik-Kommission Uniklinik RWTH Aachen, Germany, IORG0006299) with the approval number EK 062/14 and was registered (NCT02142166). Data from all patients with spontaneous SAH admitted to our institution between January 2014 and January /2020 and meeting the following inclusion criteria were prospectively recorded: (1) patient age greater than 18 years, (2) SAH verified by $\mathrm{CT}$ scan or lumbar puncture when imaging was inconclusive, (3) Hunt and Hess 1-3 on admission, and (4) diagnosis or exclusion of a bleeding source was performed by digital subtraction angiography (DSA) including a 3D rotational run. Patients with traumatic SAH, SAH from other, non-aneurysmal vascular pathologies, or with intracerebral or subdural hemorrhage were excluded.

Written informed consent was acquired from all patients or their respective legal representatives. For balanced comparison, we only included aSAH patients with milder clinical presentation (Hunt and Hess 1-3) as patients with nonaneurysmal SAH present in the majority of cases with a good/mildly impaired neurological status (Hunt and Hess 1-3). Patients without evidence of a cerebral aneurysm were further stratified into two groups according to blood distribution. Hemorrhages restricted to the interpeduncular cistern with or without extension to the ambient, chiasmatic, and horizontal part of the Sylvian cistern were classified as pmSAH. Hemorrhage patterns with extension to the interhemispheric cisterns, convexity or focus in a different location than the interpeduncular cistern and with extension to the vertical portion of the Sylvian cistern were classified as npmSAH [13, 20, 23].

\section{SAH management algorithm}

If an aneurysmal bleeding source was identified, it was secured within $48 \mathrm{~h}$ by either clipping or coiling following an interdisciplinary evaluation. All patients were closely monitored on a designated neurointensive care unit. Treatment of patients was conducted according to our local institutional standard operating procedure and has been described in detail elsewhere [27]. Acute hydrocephalus was addressed by insertion of an external ventricular drain. In patients, where clinical examination was precluded due to secondary clinical deterioration, regional neuromonitoring probes for cerebral microdialysis and brain tissue oxygen were inserted.

All patients with angiogram-negative SAH including 3D rotational reconstruction underwent early MRI scanning (cranium and cervical spine) to exclude other intra- or extraparenchymal sources of bleeding (i.e., cavernoma, fistula). In cases of a non-perimesencephalic blood distribution, an additional DSA was performed after 1 to 2 weeks to exclude an obscured vascular pathology.

\section{Data collection}

All demographic and clinical data were collected prospectively. Blood samples and blood gas analysis within $24 \mathrm{~h}$ of admission were recorded, including leucocytes, electrolytes (sodium, potassium), renal panels (creatinine, glomerular filtration rate), glucose metabolism (glucose, lactate, glucose-potassium ratio), and C-reactive protein for inflammation.

\section{Radiological, metabolic, and clinical definitions}

Primary outcome was defined as neurological outcome assessed after 6 months using the modified Rankin scale (mRS) during regular follow-ups or a structured telephone interview by a blinded investigator. The $\mathrm{mRS}$ scale was dichotomized into favorable (mRS 0-2) and unfavorable (mRS 3-6) outcome. The secondary outcomes were as follows: acute hydrocephalus, need for permanent CSF diversion (i.e., VP shunt), length of stay, DCI, DCI-related infarction, other cerebral infarction (e.g., intervention related), and mortality.

Delayed cerebral ischemia (DCI) was diagnosed according to the definition of Vergouwen et al. [25]: new focal neurological deficit or decrease in Glasgow coma scale $\geq 2$ for a duration $\geq 1 \mathrm{~h}$ or reversible after treatment and not ascribable to other reasons (e.g., infection, hydrocephalus). This definition was expanded by functional parameters in analgosedated patients, who were neurologically not assessable: characteristic hypoperfusion on $\mathrm{CT}$ perfusion, usually triggered by oxygenation crisis $\left(\mathrm{p}_{\mathrm{ti}} \mathrm{O}_{2}<10 \mathrm{mmHg}\right)$, and metabolic derangement as determined by cerebral microdialysis (lactate/pyruvate ratio $\geq 40$ ). DCI-related infarction was diagnosed either on MRI or CT scan during hospitalization. 


\section{Statistical analysis}

Quantitative parameter values are presented as median [1. quartile - 3. quartile]. Normal distribution was tested using the Shapiro-Wilk and Kolmogorov-Smirnov normality tests. Differences between two groups were analyzed using the two-sided Student's $t$-test or Mann-Whitney $U$ test. Categorical variables were analyzed in contingency tables using the chi-square test resp. Fisher's exact test. Comparisons between three groups were calculated with ANOVA or the Kruskal Wallis test, followed by Tukey's multiple comparison test or Dunn's multiple comparison test as appropriate.

Results are reported by $p$-values, odds ratio (OR), and 95\% confidence intervals (CIs) where appropriate. For all comparisons, alpha level for statistical significance was set to 0.05 . All analyses were performed using Numbers $®$, Apple Inc., Cupertino, USA, and GraphPad Prism ${ }^{\circledR}$, GraphPad Software, Inc., La Jolla, USA.

\section{Results}

\section{Baseline demographics and serum analysis for all SAH types}

Out of the total of $221 \mathrm{SAH}$ patients admitted to our hospital during the study period, 115 patients with good-grade aSAH
(Hunt and Hess 1-3) were included (Fig. 1). These patients were compared with $35 \mathrm{SAH}$ patients where no bleeding source could be identified (pmSAH: $n=16,46 \%$; npmSAH: $n=19,54 \%$ ). Demographic data are depicted in Table 1. All patients with npmSAH and pmSAH had a good grade on admission (Hunt and Hess 1-3) without statistical differences between all groups $(p=0.230)$. Modified Fisher scale was lowest in pmSAH patients, but comparable between aSAH and npmSAH $(p=0.2727)$. Patients with npmSAH were significantly older than patients with pmSAH. Smoking as an important vascular risk factor was highest in aSAH $(p=0.0028)$ without differences between npmSAH and pmSAH (see Table 1 and suppl.). Absolute serum glucose and rate of hyperglycemia on admission (glucose $>140 \mathrm{md} /$ dl) was highest in the npmSAH group (156 [134-189] mg/ $\mathrm{dl}$, resp. $72 \%$ hyperglycemia), while no patient in pmSAH presented with hyperglycemia (see Table 2 and suppl.). Likewise, serum glucose/potassium ratio showed significant differences between all groups $(p=0.0001)$ with again highest values in the npmSAH group (41.5 [32-48.25]mg/ dl). Renal function parameters were significantly elevated in the npmSAH group compared to aneurysmal SAH (creatinine, $p=0.0029$; glomerular filtration rate, $p=0.044$ ) (see Table 2 and suppl.).

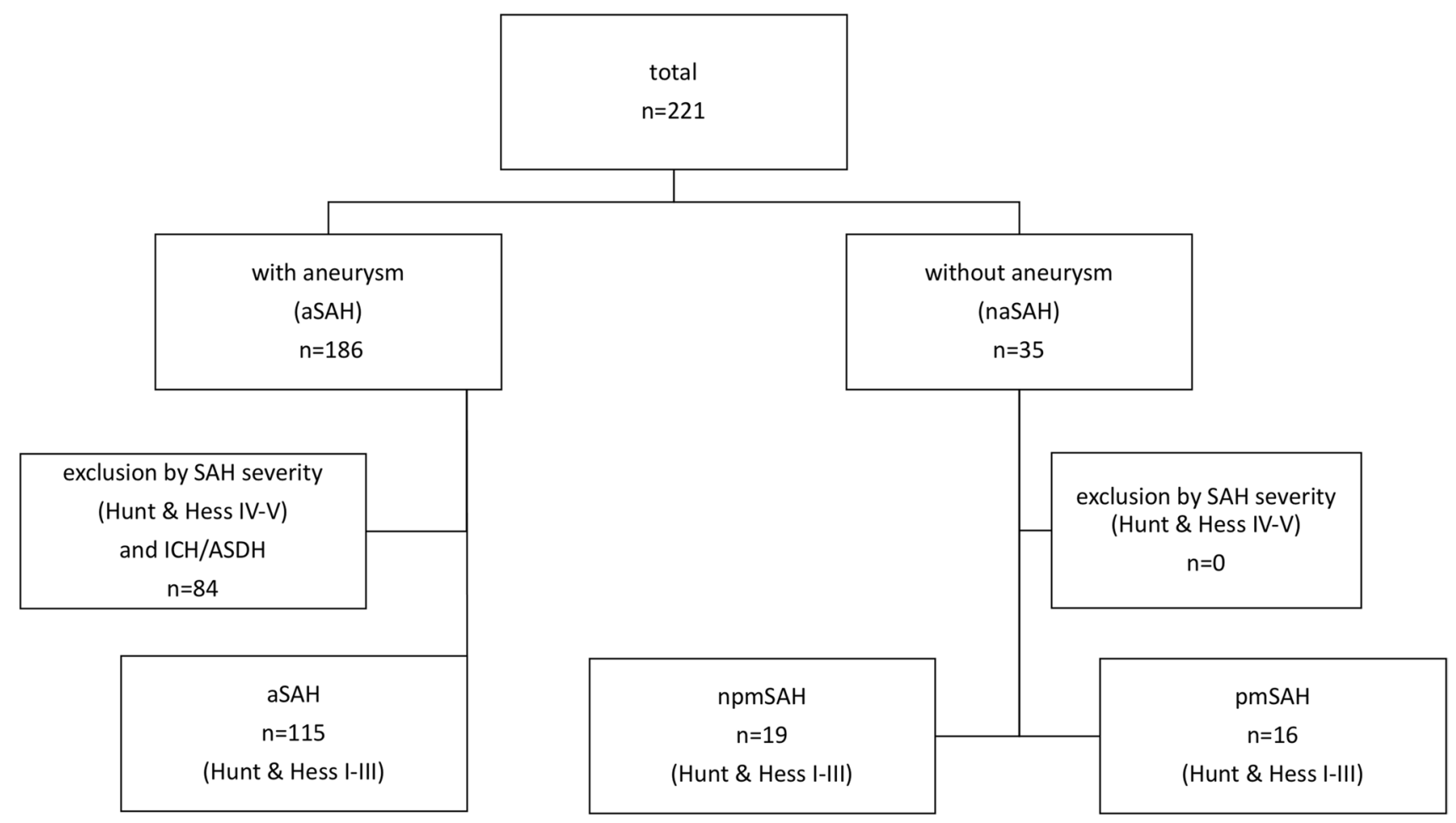

Fig. 1 Flowchart of prospective cohort enrolment. In naSAH, no patient met the exclusion criteria Hunt and Hess 4 and 5, ASDH, or ICH. na, non-aneurysmal; npm, non-perimesencephalic; pm, perimesencephalic; ICH, intracerebral hemorrhage; aSDH, acute subdural hematoma 


\section{Clinical and radiological course and outcomes for all SAH types}

Follow-up assessment after 6 months was completed in the majority of patients in all groups (see Table 3 ). Favorable outcome was reported in 13 of 14 pmSAH patients $(92.9 \%)$ and 15 of 18 npmSAH patients $(83.3 \%)$, whereas in the aSAH group, only 64 of $102(62.7 \%)$ achieved a favorable outcome $(p=0.0264)$. However, multiple comparison showed no differences between groups $(p=0.11)$ (see
Table 1 Baseline demographics, imaging features, and past medical history. $a S A H$ aneurysmal subarachnoid hemorrhage, npmSAH nonperimesencephalic $\mathrm{SAH}, p m$ perimesencephalic SAH; $p$-value, ANOVA/KruskalWallis test/chi-square as appropriate (see Supplement material for multiple comparisons)

\begin{tabular}{|c|c|c|c|c|}
\hline Parameter & aSAH & npmSAH & pmSAH & $p$-value \\
\hline Total $[n]$ & 115 & 19 & 16 & \\
\hline Age [yrs] & $55[48-65]$ & $60[56-68]$ & $52[42-60]$ & 0.032 \\
\hline Female sex $[n]$ & $75(65.2 \%)$ & $9(47.4 \%)$ & $6(37.5 \%)$ & 0.0512 \\
\hline Hunt and Hess [grade] & & & & 0.230 \\
\hline I & $33(28.6 \%)$ & $6(31.6 \%)$ & $9(56.3 \%)$ & \\
\hline II & $43(37.4 \%)$ & $8(42.1 \%)$ & $7(43.7 \%)$ & \\
\hline III & $39(33.9 \%)$ & $5(26.3 \%)$ & $0(0 \%)$ & \\
\hline Modified Fisher grade & $2[1-3]$ & $1[1,2]$ & $1[1]$ & 0.0005 \\
\hline Loss of consciousness [n] & $38 / 115(33 \%)$ & $0(0 \%)$ & $0(0 \%)$ & 0.0004 \\
\hline \multicolumn{5}{|c|}{ Past medical history and risk factors } \\
\hline Antiplatelet treatment [n] & $18(15.7 \%)$ & $3(15.8 \%)$ & $2(10.5 \%)$ & 0.946 \\
\hline Anticoagulation treatment (n) & $5(4.4 \%)$ & $2(10.5 \%)$ & $0(12.5 \%)$ & 0.32 \\
\hline Arterial hypertension [n] & $55(47.8 \%)$ & $12(63.2 \%)$ & $7(50 \%)$ & 0.415 \\
\hline Smoking & $49(42.6 \%)$ & $3(15.8 \%)$ & $1(6.25 \%)$ & 0.0028 \\
\hline
\end{tabular}

\begin{tabular}{lllll}
\hline Parameter & aSAH & npmSAH & pmSAH & $p$-value \\
\hline Total $[n]($ admission $\leq 24 \mathrm{~h})$ & $93(80.9 \%)$ & $18(94.7 \%)$ & $13(81.3 \%)$ & 0.33 \\
White blood cell count $[1000 / \mu \mathrm{l}]$ & $13[9.85-15.85]$ & $10[7.83-16.33]$ & $11.3[9.3-14.5]$ & 0.229 \\
Potassium [mmol/l] & $4.0[3.6-4.2]$ & $3.9[3.8-4.2]$ & $3.9[3.7-4.1]$ & 0.83 \\
Glucose/potassium ratio & $34[27-40]$ & $41.5[32-48.25]$ & $28[26-30]$ & $\mathbf{0 . 0 0 0 3}$ \\
Glucose $[\mathrm{mg} / \mathrm{dl}]$ & $133.0[110-157]$ & $156[134-189]$ & $108[95-120]$ & $<\mathbf{0 . 0 0 0 1}$ \\
Glucose $>140 \mathrm{mg} / \mathrm{dl}$ & $39(41.9 \%)$ & $13(72 \%)$ & $0(0 \%)$ & $\mathbf{0 . 0 0 0 3}$ \\
Creatinine $[\mathrm{mg} / \mathrm{dl}]$ & $0.7[0.6-0.8]$ & $0.82[0.74-1.0]$ & $0.78[0.63-0.96]$ & $\mathbf{0 . 0 0 1 9}$ \\
Glomerular filtration rate [ml/min] & $99.8[87.7-107.3]$ & $86.9[71.3-100.4]$ & $93.4[84.2-109.2]$ & $\mathbf{0 . 0 4 8}$ \\
C-reactive protein $[\mathrm{mg} / \mathrm{l}]$ & $2.7[1.25-4.5]$ & $2.6[0.7-3.13]$ & $2.15[1.5-5.6]$ & 0.48 \\
Lactate [mmol/l] & $1.5[1.0-2.3]$ & $1.7[1.4-2.8]$ & $1.6[0.8-1.9]$ & 0.21 \\
\hline
\end{tabular}

Table 2 Serum biomarkers on admission (patients only included when admission $\leqq$ after ictus). $a S A H$ aneurysmal subarachnoid hemorrhage, $n p m S A H$ nonperimesencephalic SAH, pm perimesencephalic SAH; ANOVA/Kruskal-Wallis test/ chi-square as appropriate. For multiple comparisons, please see the supplementary material spinal fluid, $V P$ ventriculoperitoneal, $m R S$ modified Rankin Scale. ANOVA/Kruskal-Wallis test/chi-square as appropriate. For multiple comparisons, please see the supplementary material

Table 3 Clinical and radiological outcomes. $a S A H$ aneurysmal subarachnoid hemorrhage, npmSAH non-perimesencephalic SAH, pm perimesencephalic SAH, DCI delayed cerebral ischemia, CSF cerebro-

\begin{tabular}{|c|c|c|c|c|}
\hline Parameter & aSAH & npm SAH & pmSAH & $p$-value \\
\hline DCI $[n]$ & $44(38.3 \%)$ & $7(36.8 \%)$ & $1(6.25 \%)$ & 0.041 \\
\hline DCI-related infarction $[n]$ & $18(15.7 \%)$ & $2(10.5 \%)$ & $0(0 \%)$ & 0.2095 \\
\hline Any infarction $[n]$ & $48(43.6 \%)$ & $4(21.1 \%)$ & $0(0 \%)$ & 0.0011 \\
\hline Acute hydrocephalus $[n]$ & $76(66.1 \%)$ & $14(73.7 \%)$ & $0(0 \%)$ & $<0.0001$ \\
\hline $\begin{array}{l}\text { Need for permanent CSF diversion (VP } \\
\text { shunt) }[n]\end{array}$ & $21(18.3 \%)$ & $5(26.3 \%)$ & $0(0 \%)$ & 0.106 \\
\hline Length of stay [d] & $21[17-35]$ & 19 [14-29] & $8[6-10]$ & $<0.0001$ \\
\hline In-house mortality $[n]$ & $14(12.2 \%)$ & $1(5.3 \%)$ & $0(0 \%)$ & 0.173 \\
\hline $\operatorname{mRS} 0-2$ at $6 \mathrm{mo}[n]$ & $64 / 102(62.7 \%)$ & $15 / 18(83.3 \%)$ & $13 / 14(92.9 \%)$ & 0.0264 \\
\hline
\end{tabular}


Fig. 3 and suppl.). Patients with pmSAH had the shortest hospital stay ( $8[6-10]$ days, $p<0.0001)$, while it was comparable for patients with aSAH and npmSAH (21 [17-35] days vs. 19 [14-29] days, $p=0.691$ ) (see Fig. 2 and suppl.). Rate of acute hydrocephalus and need for permanent CSF diversion were also comparable for aSAH and npmSAH patients $(66.1 \%$ vs. $73.7 \%$, OR $0.696,95 \%$ CI $0.234-2.074, p=0.61$, resp. $26.3 \%$ vs. $18.3 \%$, OR 0.626 , 95\% CI $0.21-1.93, p=0.53$ ). In contrast, no acute hydrocephalus occurred in the pmSAH group (aSAH vs. pmSAH OR $63.91,95 \%$ CI $3.7-1094, p<0.0001$; npmSAH vs. pmSAH OR 76.0, 95\% CI 4.417-1774, $p<0.0001$ ) (Fig. 2 and suppl.). The rate of DCI events was comparable in the npmSAH and aSAH group (38.5\% vs. $36.8 \%$, OR 1.06 , $95 \%$ CI $0.389-2.9, p=1.0$ ), while there was only one DCI event in the pmSAH group (aSAH vs. pmSAH OR 9.3, 95\% CI 1.19-72.9, $p=0.011$, npmSAH vs. pmSAH OR 8.75, 95\% CI 0.941-81, $p=0.047$ resp.) (see Fig. 2 and suppl.). Accordingly, DCI-related infarction rate was also comparable between aSAH and npmSAH patients ( $15.7 \%$ vs. $10.5 \%$, OR 1.6, 95\% CI 0.33-7.42, $p=0.74$ ) (see Fig. 2). Overall infarction rate (any etiology) was highest in patients with aSAH $(n=27,46.6 \%)$ without statistical difference to npmSAH $(n=4,23.5 \%)$ (OR $2.8,95 \%$ CI $0.824-9.72$, $p=0.103$ ), while cerebral infarction was absent in patients with pmSAH. In-house mortality was also absent only in the pmSAH group but failed to reach statistical significance (aSAH vs. npmSAH OR $2.5,95 \%$ CI $0.69-20.18, p=0.69$, pmSAH vs. npmSAH, OR $2.795 \%$ CI $0.1-70.4, p=1.0$ ) (see Table 3).
Fig. 2 Clinical course and complications of the different SAH entities. A Rate of acute hydrocephalus (in percentage) was comparable between aSAH and npmSAH, but significantly lower in pmSAH. B Length of stay was longest in aSAH, but comparable to npmSAH (boxwhisker, min-max). C Rate of DCI (in percentage) was comparable between aSAH and npm$\mathrm{SAH}$, but lowest in pmSAH. D DCI-related infarction (in percentage) showed no differences between groups though missing in pmSAH. aSAH, aneurysmal subarachnoid hemorrhage $(n=115)$; npmSAH, non-perimesencephalic $\mathrm{SAH}$ $(n=19)$; pmSAH, perimesencephalic SAH $(n=16)$; DCI, delayed cerebral ischemia
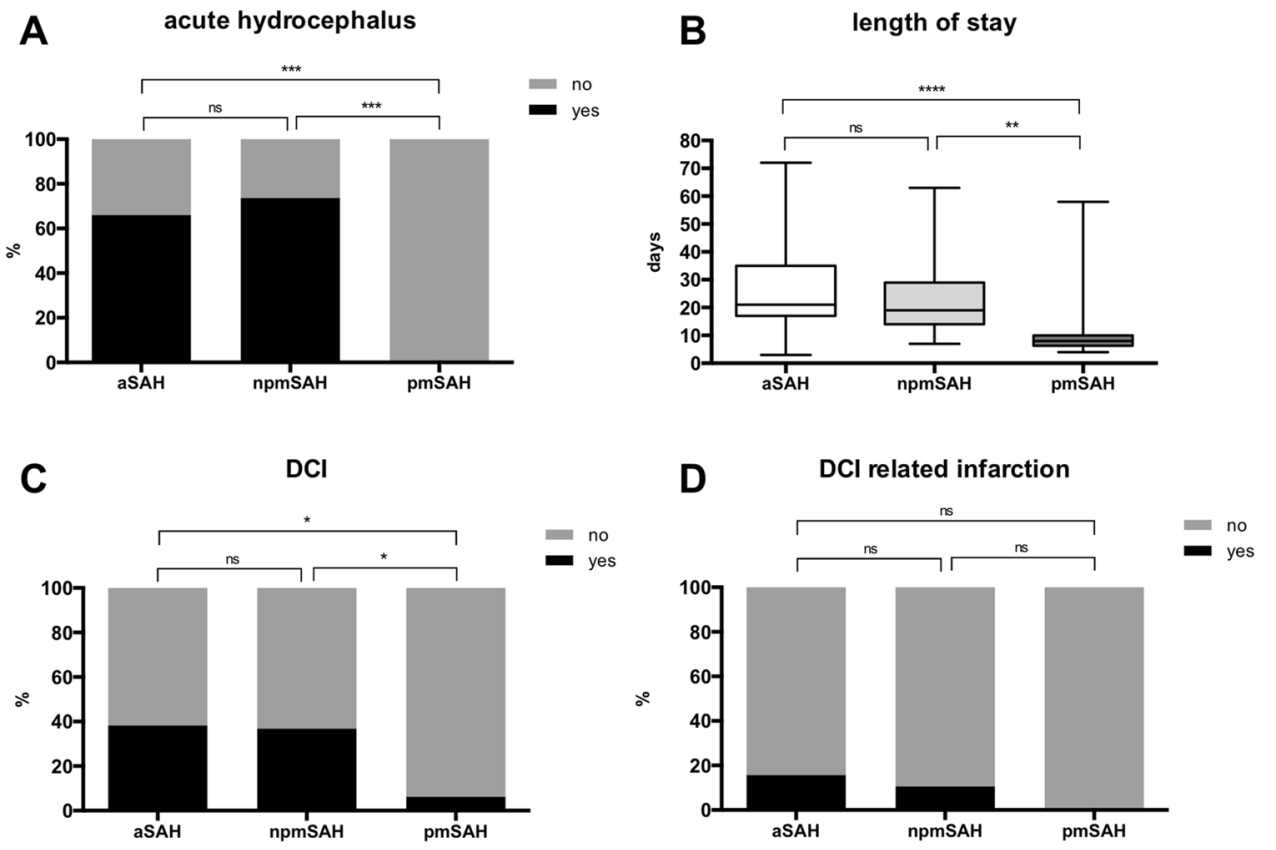

Outcome after 6 months (mRS)

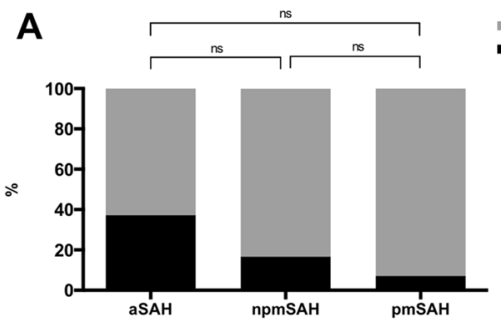

Fig. 3 Neurological outcome after 6 months. A Dichotomized neurological outcome after 6 months is depicted for aSAH $(n=102)$, npmSAH $(n=18)$, and pmSAH $(n=14)$ (good=modified Rankin scale $(\mathrm{mRS}) 0-2$, poor $=\mathrm{mRS} 3-6)$. Absolute numbers of poor outcome are highest in aSAH and lowest in pmSAH, but differences are not statis-

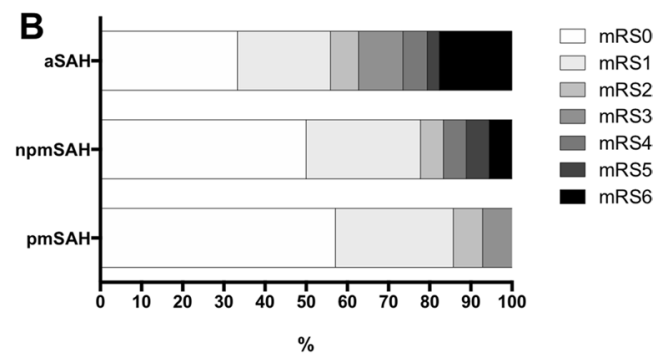

tically significant in multiple comparison test. B All mRS grades for all groups without dichotomization. aSAH, aneurysmal subarachnoid hemorrhage; npmSAH, non-perimesencephalic SAH; pm, perimesencephalic SAH 


\section{Discussion}

Non-aneurysmal subarachnoid hemorrhage is generally considered to follow a significantly more benign course with a better neurological outcome compared to aneurysmal SAH $[2,10]$. Our data, however, highlights that patients with non-aneurysmal npmSAH carry a high risk for complications, such as DCI, DCI-related infarction, hydrocephalus, and poor neurological outcome, comparable to that of good-grade aneurysmal SAH (HH 1-3) patients. Patients with characteristic pmSAH, however, generally face a more benign clinical course, rarely with complications such as hydrocephalus, DCI, or DCI-related infarction. Such patients can be discharged earlier and usually have an excellent neurological outcome.

Previous studies on aneurysmal or non-aneurysmal SAH reported conflicting results regarding baseline characteristics and clinical course. A retrospective study on 154 nonaneurysmal SAH patients highlighted that elevated CRP and white blood cell count can predict a poor neurological outcome in patients with non-aneurysmal SAH [22]. In contrast, other retrospective studies suggested a significantly aggravated clinical course only to aneurysmal SAH [15, 17] but these cohorts consisted of aSAH of all clinical grades. A recent prospective study performed by Al Mufti et al. reported a lower risk for vasospasm but comparable risk for poor outcome and DCI for npmSAH compared to aSAH confirming our own results [1]. In our study, patients with pmSAH had a more benign clinical course. These results are in line with a recently published review by Mensing et al. who showed a minimal complication rate with no deaths and rebleeding events by analyzing 208 papers [16].

Our study comprises a prospective cohort of different SAH entities that was balanced for good neurological grade on admission. There are different explanations for our findings: first, npmSAH involves the complete basal cisterns and/or subarachnoid hemorrhage space and may thereby exert its detrimental effect in a similar fashion, compared to aSAH. Although there is no evident rupture of a radiologically detectable aneurysm, the pathological cascades leading to DCI and poor neurological outcome seem to be at least partly similar. The comparable subarachnoid blood load between aSAH and npmSAH (mod Fisher scale) in our cohort may support this theory. For pmSAH, conversely, where the subarachnoid blood load is lower and more localized, numerous smaller studies suggest various venous abnormalities as bleeding origin (for review see [11] and [16]). However, interpretation of the current literature is impeded because detailed differentiation of the entities pmSAH and npmSAH has not been performed. Given the important role of early brain injury for the development of complications subsequent to aSAH, it seems reasonable to assume different pathogeneses between all three entities, and furthermore, that pmSAH and npmSAH are separate entities of diverging severity. In an experimental setting, we recently showed that the bleeding velocity itself (as assumed to be lower in the presence of a venous bleeding source) has an impact on disturbed cerebral autoregulation and pronounced early neuronal cell loss [5]. However, the bleeding source in npmSAH and pmSAH remains unknown to date, and therefore, the true impact of the bleeding event is cryptic. Second, neurological outcome assessed by the mRS was predominantly favorable in all groups, but only in pmSAH all patients achieved excellent outcome, while 37\% of good-grade aSAH and $17 \%$ of npmSAH patients had an unfavorable outcome, underlining the importance of close clinical observation in those groups (see Fig. 3).

Third, the dysregulation of glucose metabolism in the npmSAH group could also be an indicator for a more severe clinical course as already described for aSAH [4, 8, 9]: higher serum glucose variability and hyperglycemia on admission-possibly as a consequence of the stress reaction triggered by the hemorrhage-are associated with poor outcome [14]. These variables are also associated with clinical severity and thus the extent of stress hormone release and complication rate after aSAH [7, 18]. Data on glucose dynamics after non-aneurysmal SAH, however, are sparse. In our study, patients with npmSAH showed significantly higher overall glucose levels and more often presented with hyperglycemia on admission, thus possibly indicating a more severe clinical course. As npmSAH patients tended to be somewhat older in our cohort, a higher prevalence of previously undetected diabetes mellitus respectively impaired glucose tolerance may partially explain these findings. Also, hyperglycemia and higher variability in the above-mentioned studies on aSAH were primarily associated with disease severity, which in turn is a major determinant for outcome. Therefore, glucose levels may be less affected in cohorts limited to only good-grade SAH patients like ours. However, the interpretation of these findings is difficult and remains speculative in absence of serum cortisol profiles [26].

The strength of our study is the prospective design and an adequate sample of good-grade aSAH patients. However, there are also distinct limitations: the comparatively small number of patients in the pmSAH and npmSAH group is a limitation to our analysis, possibly owing to the prospective nature of our study and the comparatively long timeframe needed for recruitment. Additionally, the study lacks statistical power to provide absolute risk ratios and precluded a more rigorous matching process. Therefore, the study is not powered to detect a clinically meaningful difference in DCI rates between aSAH, pmSAH, and npmSAH. Especially the low number of patients with pmSAH $(n=16)$ precludes generalization of observed DCI rates, deaths, or treatment for hydrocephalus in this patient population. Our results should 
therefore be considered as rather hypothesis-generating and remain to be validated in a greater patient population. Additionally, we have not performed MRI scans in the acute phase in every SAH patient which would allow us a more detailed analysis of the subarachnoid blood clot distribution in relation to neuroanatomical structures between all entities. Neurological outcome assessment was performed after 6 months, which is a comparatively short time period for SAH, and the chosen outcome scale (mRS) is designed for daily routine performance and may miss more complex cognitive impairments.

In summary, our study is the first prospective analysis to relate both non-aneurysmal, non-perimesencephalic, and perimesencephalic bleeding patterns, with a balanced cohort of good-grade aSAH patients. Whether npmSAH and its aggravated clinical course is truly a separate entity with a different underlying pathophysiology remains to be determined.

We hypothesize that a more specific classification of these diverging entities is mandatory, while a pooling of these patients into a supposedly more benign group ("non-aneurysmal") should be avoided. Frequent complications such as DCI and DCI-related infarction in patients with npmSAH once more emphasize the importance of close observation not only of patients with ruptured aneurysms, but also of patients with non-aneurysmal, but also non-perimesencephalic bleeding pattern.

As a consequence, we have adopted these findings into our daily practice and have extended our standard operating procedure for aSAH towards patients with npmSAH. Patients with respective bleeding patterns remain on our ICU on average at least 14 days in order to enable timely detection of complications, while transfer to the regular ward and early discharge is encouraged in pmSAH patients.

\section{Conclusion}

In a prospective cohort of good-grade SAH patients, npmSAH and aSAH featured a similar though aggravated clinical course, advocating for prolonged neurological observation. Complications were largely absent in patients with classic pmSAH implying fundamental differences in pathophysiology and underscoring the need for differentiation of terminology and treatment in non-aneurysmal hemorrhage.

\footnotetext{
Abbreviations CT: Computed tomography; DCI: Delayed cerebral ischemia; DSA: Digital subtraction angiography; EVD: External ventricular drainage; GCS: Glasgow coma scale; HH: Hunt and Hess grade; ICP: Intracranial pressure; ICH: Intracerebral hemorrhage; MRI: Magnetic resonance imaging; ModFish: Modified Fisher scale; mRS: Modified Rankin scale; $\mathrm{p}_{\mathrm{ti}} \mathrm{O}_{2}$ : Partial pressure of brain tissue oxygen; aSAH: Aneurysmal subarachnoid hemorrhage; naSAH: Non-aneurysmal subarachnoid hemorrhage; npmSAH: Non-perimesencephalic
}

subarachnoid hemorrhage; pmSAH: Perimesencephalic subarachnoid hemorrhage; aSDH: Acute subdural hematoma; VP shunt: Ventriculoperitoneal shunt

Supplementary Information The online version contains supplementary material available at https://doi.org/10.1007/s10143-021-01650-x.

Author contribution The design and conception of this trial was developed by CC and GS. CC, MW, KS, TS, and WA were involved in data acquisition. Statistical analysis was performed by CC. All authors were involved in the interpretation of data. The manuscript was drafted and illustration was created by CC. All authorship requirements have been met, and the final manuscript was critically revised and approved by all authors.

Funding Open Access funding enabled and organized by Projekt DEAL.

Data availability The raw data of this analysis can be made available by the authors to any qualified researcher.

Code availability Not applicable.

\section{Declarations}

Ethics approval This study has been approved by the local ethics committee (Ethikkommission RWTH Aachen). We certify that the study was performed in accordance with the ethical standards as laid down in the 1964 Declaration of Helsinki and its later amendments or comparable ethical standards. This manuscript is written and composed according to the STROBE statement for reporting of observational studies.

Consent to participate Written informed consent was acquired from all individual patients or their respective legal representatives.

Consent for publication Patients signed informed consent regarding publishing their anonymized data.

Conflict of interest The authors declare no competing interests.

Open Access This article is licensed under a Creative Commons Attribution 4.0 International License, which permits use, sharing, adaptation, distribution and reproduction in any medium or format, as long as you give appropriate credit to the original author(s) and the source, provide a link to the Creative Commons licence, and indicate if changes were made. The images or other third party material in this article are included in the article's Creative Commons licence, unless indicated otherwise in a credit line to the material. If material is not included in the article's Creative Commons licence and your intended use is not permitted by statutory regulation or exceeds the permitted use, you will need to obtain permission directly from the copyright holder. To view a copy of this licence, visit http://creativecommons.org/licenses/by/4.0/.

\section{References}

1. Al-Mufti F, Merkler AE, Boehme AK, Dancour E, May T, Schmidt JM, Park S, Connolly ES, Lavine SD, Meyers PM et al (2018) Functional outcomes and delayed cerebral ischemia following nonperimesencephalic angiogram-negative subarachnoid hemorrhage similar to aneurysmal subarachnoid hemorrhage. Neurosurgery 82(3):359-364 
2. Alhoobi M, Abu-Qadous F, Khan M, Shaaban A, Shaikh N, Hammadi F, Abu-Jarir R, Albanna W, Alrumaihi G, Belkhair $S$ et al (2020) Ten years' experiences in the treatment of nonaneurysmal subarachnoid hemorrhage: a retrospective analysis of outcome parameters in a single-center study. Asian J Neurosurg 15(2):315-321

3. Buyukkaya R, Yildirim N, Cebeci H, Kocaeli H, Dusak A, Ocakoglu G, Erdogan C, Hakyemez B (2014) The relationship between perimesencephalic subarachnoid hemorrhage and deep venous system drainage pattern and calibrations. Clin Imaging 38(3):226-230

4. Chou SH, Robertson CS (2014) Participants in the International Multi-disciplinary Consensus Conference on the Multimodality M: Monitoring biomarkers of cellular injury and death in acute brain injury. Neurocrit Care 21(Suppl 2):S187-214

5. Conzen C, Becker K, Albanna W, Weiss M, Bach A, Lushina N, Steimers A, Pinkernell S, Clusmann H, Lindauer U et al (2019) The Acute phase of experimental subarachnoid hemorrhage: intracranial pressure dynamics and their effect on cerebral blood flow and autoregulation. Transl Stroke Res 10(5):566-582

6. Elhadi AM, Zabramski JM, Almefty KK, Mendes GA, Nakaji P, McDougall CG, Albuquerque FC, Preul MC, Spetzler RF (2015) Spontaneous subarachnoid hemorrhage of unknown origin: hospital course and long-term clinical and angiographic follow-up. $\mathbf{J}$ Neurosurg 122(3):663-670

7. Frontera JA, Fernandez A, Claassen J, Schmidt M, Schumacher HC, Wartenberg K, Temes R, Parra A, Ostapkovich ND, Mayer SA (2006) Hyperglycemia after SAH: predictors, associated complications, and impact on outcome. Stroke; 37(1):199-203

8. Fujiki Y, Matano F, Mizunari T, Murai Y, Tateyama K, Koketsu K, Kubota A, Kobayashi S, Yokota H, Morita A (2018) Serum glucose/potassium ratio as a clinical risk factor for aneurysmal subarachnoid hemorrhage. J Neurosurg 129(4):870-875

9. Jabbarli R, Pierscianek D, DarkwahOppong M, Sato T, Dammann P, Wrede KH, Kaier K, Kohrmann M, Forsting M, Kleinschnitz C et al (2018) Laboratory biomarkers of delayed cerebral ischemia after subarachnoid hemorrhage: a systematic review. Neurosurg Rev 43(3):825-833

10. Kang DH, Park J, Lee SH, Park SH, Kim YS, Hamm IS (2009) Does non-perimesencephalic type non-aneurysmal subarachnoid hemorrhage have a benign prognosis? J Clin Neurosc 16(7):904-908

11. Kapadia A, Schweizer TA, Spears J, Cusimano M, Macdonald RL (2014) Nonaneurysmal perimesencephalic subarachnoid hemorrhage: diagnosis, pathophysiology, clinical characteristics, and long-term outcome. World Neurosurg 82(6):1131-1143

12. Konczalla J, Kashefiolasl S, Brawanski N, Lescher S, Senft C, Platz J, Seifert V (2016) Cerebral vasospasm and delayed cerebral infarctions in 225 patients with non-aneurysmal subarachnoid hemorrhage: the underestimated risk of Fisher 3 blood distribution. J Neurointerv Surg 8(12):1247-1252

13. Konczalla J, Platz J, Schuss P, Vatter H, Seifert V, Guresir E (2014) Non-aneurysmal non-traumatic subarachnoid hemorrhage: patient characteristics, clinical outcome and prognostic factors based on a single-center experience in 125 patients. BMC Neurol $14: 140$

14. Kruyt ND, Biessels GJ, de Haan RJ, Vermeulen M, Rinkel GJ, Coert B, Roos YB (2009) Hyperglycemia and clinical outcome in aneurysmal subarachnoid hemorrhage: a meta-analysis. Stroke 40(6): $424-430$
15. Lago A, Lopez-Cuevas R, Tembl JI, Fortea G, Gorriz D, Aparici F, Parkhutik V (2016) Short- and long-term outcomes in nonaneurysmal non-perimesencephalic subarachnoid hemorrhage. Neurol Res 38(8):692-697

16. Mensing LA, Vergouwen MDI, Laban KG, Ruigrok YM, Velthuis BK, Algra A, Rinkel GJE (2018) Perimesencephalic hemorrhage: a review of epidemiology, risk factors, presumed cause, clinical course, and outcome. Stroke 49(6):1363-1

17. Nesvick CL, Oushy S, Rinaldo L, Wijdicks EF, Lanzino G, Rabinstein AA (2019) Clinical complications and outcomes of angiographically negative subarachnoid hemorrhage. Neurology 92(20):e2385-e2394

18. Okazaki T, Hifumi T, Kawakita K, Shishido H, Ogawa D, Okauchi M, Shindo A, Kawanishi M, Tamiya T, Kuroda Y (2018) Blood glucose variability: a strong independent predictor of neurological outcomes in aneurysmal subarachnoid hemorrhage. J Intensive Care Med 33(3):189-195

19. Raya A, Zipfel GJ, Diringer MN, Dacey RG Jr, Derdeyn CP, Rich KM, Chicoine MR, Dhar R (2014) Pattern not volume of bleeding predicts angiographic vasospasm in nonaneurysmal subarachnoid hemorrhage. Stroke 45(1):265-267

20. Rinkel GJ, Wijdicks EF, Hasan D, Kienstra GE, Franke CL, Hageman LM, Vermeulen M, van Gijn J (1991) Outcome in patients with subarachnoid haemorrhage and negative angiography according to pattern of haemorrhage on computed tomography. Lancet 338(8773):964-968

21. Rouchaud A, Lehman VT, Murad MH, Burrows A, Cloft HJ, Lindell EP, Kallmes DF, Brinjikji W (2016) Nonaneurysmal perimesencephalic hemorrhage is associated with deep cerebral venous drainage anomalies: a systematic literature review and meta-analysis. AJNR Am J Neuroradiol 37(9):1657-1663

22. Schuss P, Hadjiathanasiou A, Brandecker S, Guresir A, Vatter H, Guresir E (2018) Elevated C-reactive protein and white blood cell count at admission predict functional outcome after non-aneurysmal subarachnoid hemorrhage. J Neurol 265(12):2944-2948

23. van Gijn J, van Dongen KJ, Vermeulen M, Hijdra A (1985) Perimesencephalic hemorrhage: a nonaneurysmal and benign form of subarachnoid hemorrhage. Neurology 35(4):493-497

24. van Gijn J, Rinkel GJ (2001) Subarachnoid haemorrhage: diagnosis, causes and management. Brain 124(Pt 2):249-278

25. Vergouwen MD, Vermeulen M, van Gijn J, Rinkel GJ, Wijdicks EF, Muizelaar JP, Mendelow AD, Juvela S, Yonas H, Terbrugge KG et al (2010) Definition of delayed cerebral ischemia after aneurysmal subarachnoid hemorrhage as an outcome event in clinical trials and observational studies: proposal of a multidisciplinary research group. Stroke 41(10):2391-2395

26. Vergouwen MD, van Geloven N, de Haan RJ, Kruyt ND, Vermeulen M, Roos YB (2010) Increased cortisol levels are associated with delayed cerebral ischemia after aneurysmal subarachnoid hemorrhage. Neurocrit Care 12(3):342-345

27. Weiss M, Conzen C, Mueller M, Wiesmann M, Clusmann H, Albanna W, Schubert GA (2019) Endovascular rescue treatment for delayed cerebral ischemia after subarachnoid hemorrhage is safe and effective. Front Neurol 10:136

Publisher's note Springer Nature remains neutral with regard to jurisdictional claims in published maps and institutional affiliations. 